\title{
Microdeletions in 16p11.2 and 13q31.3 associated with developmental delay and generalized overgrowth
}

\author{
A.M. George ${ }^{1}$, J. Taylor ${ }^{2}$ and D.R. Love ${ }^{1}$ \\ ${ }^{1}$ Diagnostic Genetics, LabPlus, Auckland City Hospital, Auckland, \\ New Zealand \\ ${ }^{2}$ Northern Regional Genetic Service, Auckland City Hospital, Auckland, \\ New Zealand \\ Corresponding author: D.R. Love \\ E-mail: donaldl@adhb.govt.nz
}

Genet. Mol. Res. 11 (3): 3133-3137 (2012)

Received November 28, 2011

Accepted July 18, 2012

Published September 3, 2012

DOI http://dx.doi.org/10.4238/2012.September.3.1

\begin{abstract}
Chromosome microarray analysis of patients with developmental delay has provided evidence of small deletions or duplications associated with this clinical phenotype. In this context, a 7.1 - to $8.7-\mathrm{Mb}$ interstitial deletion of chromosome 16 is well documented, but within this interval a rare $200-\mathrm{kb}$ deletion has recently been defined that appears to be associated with obesity, or developmental delay together with overgrowth. We report a patient carrying this rare deletion, who falls into the latter clinical category, but who also carries a second very rare deletion in 13q31.3. It remains unclear if this maternally inherited deletion acts as a second copy number variation leading to pathogenic variation, or is non-causal and the true modifiers are yet to be determined.
\end{abstract}

Key words: Developmental delay; Obesity; Overgrowth; GPC5; SH2B1 


\section{INTRODUCTION}

Current referrals for chromosome microarray analysis (CMA) are primarily for determining the molecular basis of developmental delay and autistic spectrum disorder in childhood. The introduction of this analysis has resulted in approximately $15 \%$ of these patients having their phenotype delineated by a copy number change (Miller et al., 2010). Frequently, the primary referral indication of developmental delay is associated with other dysmorphic features and a syndrome may be defined. The 16p11.2 microdeletion has been identified in up to $1 \%$ of autistic individuals and varies in length from a critical $600 \mathrm{~kb}$ to approximately $9 \mathrm{Mb}$ (extending into 16p12.2) (Ballif et al., 2007; Weiss et al., 2008; Kumar et al., 2008; Marshall et al., 2008; Battaglia et al., 2009; Hempel et al., 2009). The $600-\mathrm{kb}$ region is associated with a broad clinical phenotype that encompasses autism and developmental disorders.

A recently identified $200-\mathrm{kb}$ deletion of $16 \mathrm{p} 11.2$ that lies at the proximal end of the larger deletions, but distal to the $600-\mathrm{kb}$ critical region, has been described in a recent review (Bachmann-Gagescu et al., 2010; Figure 1). Patients with this 200-kb deletion exhibit severe early onset obesity or developmental delay with overgrowth. In terms of the former clinical outcome, the deletion encompasses the SH2B1 gene, which has been implicated in obesity (Bochukova et al., 2010); however, the role of this gene in patients with the latter clinical phenotype is less clear. The current case report examines a patient who has a deletion that precisely falls in the 200-kb interval, but does not display obesity (Figure 1). This patient also carries a second deletion, which lies in 13q31.3, and encompasses part of the GPC5 gene, which has not been reported to date.

\section{PATIENT, MATERIAL AND METHODS}

The patient was initially examined at 3 years and 10 months of age. He was described as 'floppy' as a baby achieving milestones late, for example walking at 2 years. He displayed significant delay in speech development and interacted poorly with other children. His height and weight were in the 75th percentile with a head circumference in the 90th percentile. He was not described as obese, his weight being proportionate to height. Dysmorphic features were mild with bilateral strabismus, extra teeth and a high narrowly arched palate. His siblings displayed possible Asperger-type behavior and the father struggled at school. The mother was noted to be short, below the 5th percentile, overweight, as were the rest of her family, and of normal intelligence.

The patient was referred for CMA analysis, and genomic DNA was purified using Puregene (Qiagen Pty Ltd.). The DNA was subjected to CMA using an Affymetrix ${ }^{\circledR}$ Cytogenetics Whole-Genome 2.7M Array according to manufacturer instructions. Regions of copy number change were determined using the Affymetrix Chromosome Analysis Suite v.1.0.1 software (ChAS), and interpreted with the aid of the UCSC Genome Browser (http://genome.ucsc.edu/; Human March 2006 (hg18) assembly). The inheritance of copy number changes identified in the patient was investigated by analyzing parental samples using Affymetrix cytogenetic arrays.

\section{RESULTS AND DISCUSSION}

CMA revealed a de novo 220-kb heterozygous deletion involving region 16p11.2 (co- 
ordinates chr 16: 28,733,674-28,949,422; Figure 1), together with a maternally inherited 240-kb heterozygous deletion of region 13q31.3 (coordinates chr13: 90,859,647-91,100,336; Figure 2).

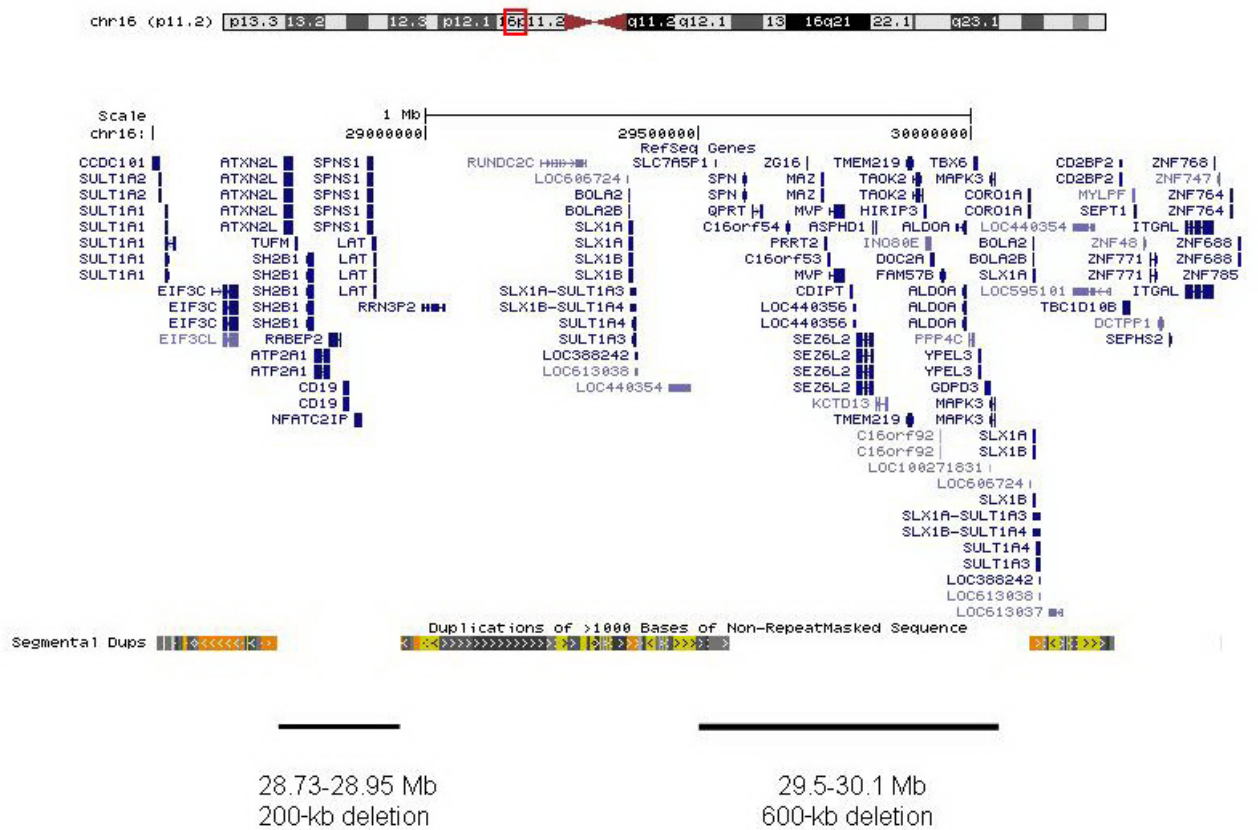

Figure 1. Location and extent of chromosome 16p11.2 deletions. A UCSC Genome Browser (March 2006 (hg18) assembly) view of the chromosomal region 16p11.2 (chr16: 28,500,000-30,500,000) is shown, together with Refseq genes and segmental duplications. The bottom panel shows the location and extent of the $200-\mathrm{kb}$ (the patient reported here) and 600-kb deletions in 16p11.2.
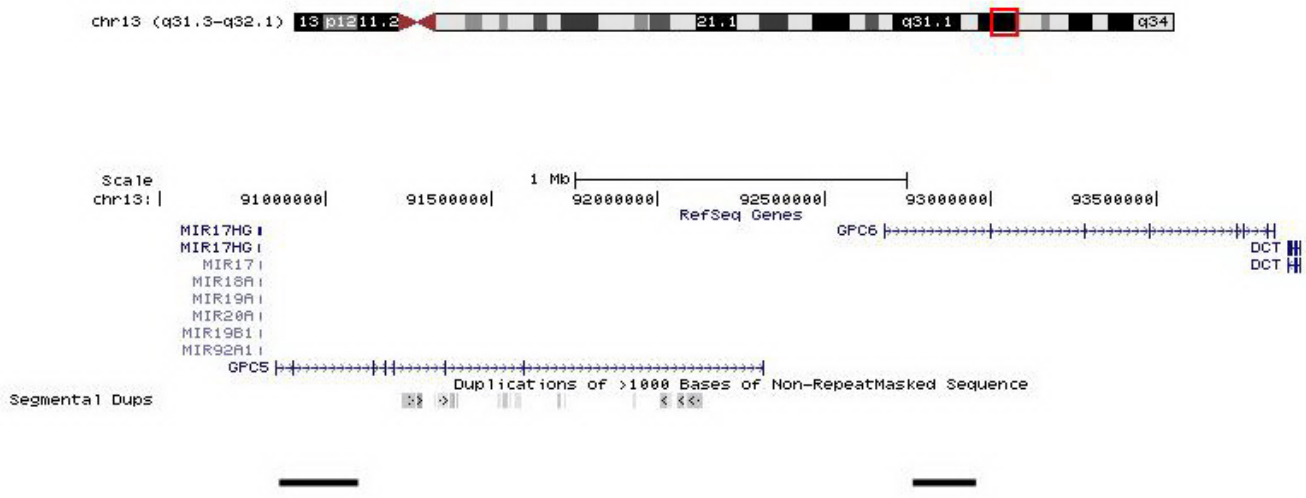

$90.86-91.10 \mathrm{Mb}^{*}$

$92.88-93.04 \mathrm{Mb}$

Figure 2. Location and extent of chromosome 13q31.3 deletions. A UCSC Genome Browser (March 2006 (hg18) assembly) view of the chromosomal region 13q31.3 (chr13: 90,500,000-94,000,000) is shown, together with FISH clones, Refseq genes and segmental duplications. The bottom panel shows the location and extent of the $13 \mathrm{q} 31.3$ deletion in the patient described here (indicated by an asterisk), and of the case reported by Bachmann-Gagescu et al. (2010). 
The 16p11.2 region overlaps with the proximal end of the 16p11.2-p12.2 microdeletion syndrome, but constitutes only a small part of the more commonly detected 7.1- to 8.7-Mb deletion (Ballif et al., 2007; Hempel et al., 2009; Battaglia et al., 2009). The 16p11.2 region is a 'hot spot' for recombination events due to the presence of segmental duplications, and hence a high likelihood of nonallelic homologous recombination. Figure 1 shows the location of segmental duplications that immediately flank the $220-\mathrm{kb}$ deletion interval.

Genes located in the 220-kb region include SH2B1 (OMIM \#608937), which plays a role in body fat leptin and insulin signaling (Bachmann-Gagescu et al., 2010). A brief review of the literature and the DECIPHER database (http://decipher.sanger.ac.uk/) shows 14 patients with the 200-kb deletion in 16p11.2 but with the common feature of developmental delay, intellectual disability or cognitive deficiency (Bijlsma et al., 2009; Firth et al., 2009; BachmannGagescu et al., 2010; Bochukova et al., 2010; Mefford et al., 2010). Half of the patients were obese; however, three of these were from a biased study targeting early onset obesity (Bochukova et al., 2010); 5/14 had a height $>75$ th percentile. The other 16p11.2-localized genes deleted in our case included RAB3P2, ATXN2L, TUFM, SPNS1, ATP2A1, LAT, NFATC2IP, and $C D 19$, none of which appear to be implicated in the phenotype.

The 16p deletion event may be a risk factor that acts in concert with a second factor to give rise to variation in the severity of the disease. The 'second hit' could be either another copy number variation (CNV; an abnormal number of copies, detected as a loss or gain, of a region of DNA), or a small disrupting mutation in a related gene, or an environmental event (Girirajan et al., 2010). This two-hit hypothesis may explain the comorbidity that exists between cognitive impairment, autism and schizophrenia, in addition to the variation in dysmorphic features associated with microdeletion/duplication syndromes (Fombonne, 2002; Font-Llitjos et al., 2005; Sharp et al., 2008; Woodberry et al., 2008).

None of the six cases described in clinical detail by Bachmann-Gagescu et al. (2010) had a second genomic imbalance; however, three of 31 cases with the 200-kb deletion did have a second $\mathrm{CNV}$ of uncertain clinical significance. In terms of the patient reported here, an intragenic deletion was detected in $13 \mathrm{q} 31.3$, which is located in the GPC5 gene, encoding for glypican 5 (OMIM \#602446). This protein is a cell surface sulfate proteoglycan that may play a role in the control of cell division and growth regulation. Deletions in this gene have not been reported in databases of normal variants. Interestingly, Bachmann-Gagescu et al. (2010) reported a patient with an intragenic deletion of $13 q 31.3(92,880,558-93,044,066)$ located within the GPC6 gene, which is located distal to that seen in this case report (Figure 2). It is tempting to speculate that these deletions contribute to variation in the $16 \mathrm{p} 11.2$ deletion phenotype. There are 6 members of the glypican family, GPC1 to GPC6. Defects in GPC5 have not been associated with disease; however, mutations in GPC3 and GPC4 cause Simpson-Golabi-Belimel syndrome, which is an X-linked overgrowth syndrome (Shi and Filmus, 2009). GPC5 and GPC6 show homology to GPC3 and GPC4. Mutations in the GPC6 gene have been implicated in omodysplasia and heart disease. Mouse models have shown expression of both GPC5 and GPC6 in developing limbs (Quélin et al., 2009). Our patient exhibits generalized overgrowth and developmental delay that agrees with the findings of Bachmann-Gagescu et al. (2010), but is in contrast to the severe obesity noted in the cases reported by Bochukova et al. (2010).

This case adds to the evidence for a phenotype of developmental delay, generalized overgrowth, with or without obesity, together with minor dysmorphic features that are associated with deletion events in 16p11.2, but possibly involving other loci. The 13q deletion of unknown clinical significance may be acting as a modifier. In this case, the mother 
carries the $13 \mathrm{q}$ deletion event and is short and obese, but the proband is tall and of normal weight. The alternative explanation is that the 16p11.2 deletion event underlies the obesity/ over-growth phenotype, but other as yet to be determined genetic factors play a role in developmental delay.

\section{ACKNOWLEDGMENTS}

We acknowledge the assistance of Fern Ashton and Jennifer Respicio in undertaking the CMA reported here.

\section{REFERENCES}

Bachmann-Gagescu R, Mefford HC, Cowan C, Glew GM, et al. (2010). Recurrent 200-kb deletions of 16p11.2 that include the SH2B1 gene are associated with developmental delay and obesity. Genet. Med. 12: 641-647.

Ballif BC, Hornor SA, Jenkins E, Madan-Khetarpal S, et al. (2007). Discovery of a previously unrecognized microdeletion syndrome of 16p11.2-p12.2. Nat. Genet. 39: 1071-1073.

Battaglia A, Novelli A, Bernardini L, Igliozzi R, et al. (2009). Further characterization of the new microdeletion syndrome of 16p11.2-p12.2. Am. J. Med. Genet. A. 149A: 1200-1204.

Bijlsma EK, Gijsbers AC, Schuurs-Hoeijmakers JH, van HA, et al. (2009). Extending the phenotype of recurrent rearrangements of 16p11.2: deletions in mentally retarded patients without autism and in normal individuals. Eur. J. Med. Genet. 52: 77-87.

Bochukova EG, Huang N, Keogh J, Henning E, et al. (2010). Large, rare chromosomal deletions associated with severe early-onset obesity. Nature 463: 666-670.

Firth HV, Richards SM, Bevan AP, Clayton S, et al. (2009). DECIPHER: Database of chromosomal imbalance and phenotype in humans using ensembl resources. Am. J. Hum. Genet. 84: 524-533.

Fombonne E (2002). Epidemiological trends in rates of autism. Mol. Psychiatry 7 (Suppl 2): S4-S6.

Font-Llitjos M, Jimenez-Vidal M, Bisceglia L, Di Perna M, et al. (2005). New insights into cystinuria: 40 new mutations, genotype-phenotype correlation, and digenic inheritance causing partial phenotype. J. Med. Genet. 42: 58-68.

Girirajan S, Rosenfeld JA, Cooper GM, Antonacci F, et al. (2010). A recurrent 16p12.1 microdeletion supports a two-hit model for severe developmental delay. Nat. Genet. 42: 203-209.

Hempel M, Rivera BN, Wagenstaller J, Lederer G, et al. (2009). Microdeletion syndrome 16p11.2-p12.2: clinical and molecular characterization. Am. J. Med. Genet. A. 149A: 2106-2112.

Kumar RA, Karamohamed S, Sudi J, Conrad DF, et al. (2008). Recurrent 16p11.2 microdeletions in autism. Hum. Mol. Genet. 17: 628-638.

Marshall CR, Noor A, Vincent JB, Lionel AC, et al. (2008). Structural variation of chromosomes in autism spectrum disorder. Am. J. Hum. Genet. 82: 477-488.

Mefford HC, Muhle H, Ostertag P, von Spiczak S, et al. (2010). Genome-wide copy number variation in epilepsy: novel susceptibility loci in idiopathic generalized and focal epilepsies. PLoS Genet. 6: e1000962.

Miller DT, Adam MP, Aradhya S, Biesecker LG, et al. (2010). Consensus statement: chromosomal microarray is a firsttier clinical diagnostic test for individuals with developmental disabilities or congenital anomalies. Am. J. Hum. Genet. 86: 749-764.

Quélin C, Bendavid C, Dubourg C, de la Rochebrochard C, et al. (2009). Twelve new patients with 13q deletion syndrome: genotype-phenotype analyses in progress. Eur. J. Med. Genet. 52: 41-46.

Sharp AJ, Mefford HC, Li K, Baker C, et al. (2008). A recurrent 15q13.3 microdeletion syndrome associated with mental retardation and seizures. Nat. Genet. 40: 322-328.

Shi W and Filmus J (2009). A patient with the Simpson-Golabi-Behmel syndrome displays a loss-of-function point mutation in GPC3 that inhibits the attachment of this proteoglycan to the cell surface. Am. J. Med. Genet. A. 149A: 552-554.

Weiss LA, Shen Y, Korn JM, Arking DE, et al. (2008). Association between microdeletion and microduplication at 16p11.2 and autism. N. Engl. J. Med. 358: 667-675.

Woodberry KA, Giuliano AJ and Seidman LJ (2008). Premorbid IQ in schizophrenia: a meta-analytic review. Am. J. Psychiatry 165: 579-587. 